\title{
DESEMPENHO DA ATENÇÃO PRIMÁRIA À SAÚDE NA PERSPECTIVA DOS PROFISSIONAIS: UMA REVISÃO INTEGRATIVA
}

\author{
PERFORMANCE OF PRIMARY HEALTH CARE FROM THE \\ PERSPECTIVE OF PROFESSIONALS: AN INTEGRATIVE REVIEW
}

Rafaela Simão de Abrantes ${ }^{1}$ Aline de Paula Rêgo Graciano Luz ${ }^{2}$

Alba Rossana Vieira Costa ${ }^{3}$ Priscilla Kelly Policarpo Falcão ${ }^{4}$

RESUMO: OBJETIVO: Realizar uma revisão integrativa da literatura a partir de artigos publicados que utilizaram o PCATool - versão profissionais, para avaliar a APS. MÉTODOS: Foram utilizados os descritores PCATooL e Primary Care Assessment Tool nas bases de dados SciElo, PubMed e Lilacs. RESULTADOS: Identificaram-se 250 artigos nacionais e internacionais, publicados nos últimos dez anos, dos quais 34 foram incluídos nessa revisão, após leitura completa e aplicação dos critérios estabelecidos. Os atributos mais bem avaliados foram o enfoque familiar, integralidade e coordenação da atenção. A acessibilidade de primeiro contato foi 0 atributo com pior desempenho. Houve predominância de estudos realizados no Brasil. CONCLUSÕES: Os resultados demonstram que existem ainda desafios para a concretização de uma atenção primária de qualidade, sobretudo na acessibilidade de primeiro contato.

Palavras chave: Atenção Primária à Saúde. Avaliação de Serviços de Saúde. Profissionais de Saúde.

ABSTRACT: OBJECTIVE: To carry out an integrative literature review based on published articles that used the PCATool - professional version, to assess PHC.

\footnotetext{
Mestranda em Saúde Pública pela Universidade Estadual da Paraíba (UEPB). E-mail: rafaelaabrantes19@gmail.com.

2 Mestranda em Saúde Pública pela Universidade Estadual da Paraíba (UEPB). E-mail: aline_grac@yahoo.com.br.

${ }^{3}$ Enfermeira Residente em UTI - COREMU/IMIP. E-mail: vieira.albarossana@gmail.com.

4 Mestranda em Saúde Pública pela Universidade Estadual da Paraíba (UEPB). E-mail: pripolicarpo@gmail.com.
} 
METHODS: The descriptors PCATooL and Primary Care Assessment Tool were used in the SciElo, PubMed and Lilacs databases. RESULTS: 250 national and international articles, published in the last ten years, were identified, of which 34 were included in this review, after a complete reading and application of the established criteria. The best evaluated attributes were the family focus, comprehensiveness and coordination of care. First contact accessibility was the attribute with the worst performance. There was a predominance of studies conducted in Brazil. CONCLUSIONS: The results demonstrate that there are still challenges for the realization of quality primary care, especially in terms of access to first contact.

Keywords: Primary Health Care. Health Services Evaluation. Health Personnel. 


\section{INTRODUÇÃO}

A Atenção Primária à Saúde (APS), definida a partir da Conferência de Alma Atta, em 1978, configurou-se como a principal estratégia de organização dos sistemas de saúde, sendo estabelecida como o primeiro nível de atenção dentro de um sistema universal, com oferta de cuidados de saúde individual e coletivo contínuos, estando integrada ao desenvolvimento social e econômico do país (OMS, 1979).

Baseado nesses princípios, Starfield sistematizou a APS em atributos essenciais e derivados. São quatro os atributos essenciais: acesso de primeiro contato - acessibilidade e utilização do serviço como primeira escolha do usuário; longitudinalidade - envolve o vínculo ao longo do tempo entre usuário e profissionais, com garantia de um cuidado acompanhado pelos mesmos profissionais; Coordenação da atenção - garantia da continuidade do tratamento nos diferentes pontos do sistema; Integralidade - oferta de serviços disponíveis para a promoção de saúde, prevenção, tratamento e reabilitação das doenças. Além destes, existem ainda três atributos derivados: orientação familiar - cuidado ofertado com enfoque familiar; orientação comunitária - prestação do cuidado de acordo com a cultura e contexto da comunidade; competência cultural - atuação profissional que leva em consideração a cultura, crenças e valores da comunidade (STARFIELD, 2002).

A partir dessa definição, foi construído o Primary Care Assessment Tool (PCATooL), por Starfield e colaboradores (2002). Esse instrumento, validado no Brasil (HAUSER et al., 2013) e em diversas nações como Taiwan, Hong Kong, Canadá, Espanha, Coreia do Sul, Argentina e Japão (TSAl et al, 2010a; TSAl et al, 2010b, WONG et al, 2010; HAGGERTY et al, 2007; PASARÍN et al, 2007; LEE et al, 2009; BERRA et al, 2011; BERRA et al, 2013,

BRESICK et al., 2015; AOKI, INOUE, NAKAYAMA, 2016), é capaz de avaliar a qualidade dos serviços de atenção primária, por meio da mensuração da presença e extensão dos atributos da APS. 
O PCATooL presenta-se originalmente nas versões usuário adulto, infantil, profissional e/ou gestores (STARFIELD, 2002), sendo conhecido no Brasil como Instrumento de Avaliação da Atenção Primária (PCATooL-Brasil) (HARZHEIM, 2006; DUNCAN, 2006; HAUSER et al., 2013). Atualmente, é considerado a ferramenta mais adequada para avaliar a atenção primária, sendo caracterizado pela sua simplicidade e aplicabilidade dos resultados dos escores pela gestão local (FRACOLLI et al., 2014; D’ÁVILLA et al., 2017).

O conhecimento do desempenho dos serviços de atenção primária, por meio de estudos avaliativos, é indispensável para a elaboração de estratégias que visem seu aperfeiçoamento e a potencialização dos aspectos positivos. No entanto, o conhecimento da perspectiva avaliativa dos profissionais, no Brasil e no mundo, não tem sido analisado em revisões, diferentemente do que ocorre com usuários (D’ÁVILLA et al., 2017), mesmo com a carga relevante desses estudos.

Considerando a relevância da avaliação em saúde para a qualificação da APS, o presente estudo teve como objetivo apresentar uma revisão integrativa de estudos que utilizaram o PCATooL-versão profissionais de saúde, realizados no Brasil e em outros países e discutir os resultados encontrados.

\section{MÉTODOS}

Para a revisão buscou-se estudos nas bases de dados Pubmed, da National Library of Medicine dos Estados Unidos, Lilacs (Literatura Latino-Americana e do Caribe em Ciências da Saúde) e SciELO (Scientific Electonic Library Online). Foram selecionados artigos publicados nos últimos dez anos (2009 a 2019). As palavras chaves utilizadas foram Primary Care Assessment Tool e PCATooL, com operador boleano "OR".

A pesquisa resultou em 250 artigos publicados. Após leitura dos resumos, foram selecionados 42 para leitura na íntegra. Os critérios de inclusão para seleção final dos artigos foram: estudos no período, nos idiomas inglês, espanhol e português e estudos do tipo transversal que utilizaram o PCATooL versão 
profissionais. Foram excluídos artigos que utilizaram versões adaptadas do PCATooL, como aquela que avalia o cuidado aos pacientes com tuberculose, estudos qualitativos, além de artigos quantitativos com amostras inferiores a 20 profissionais de saúde.

Totalizou-se 34 artigos incluídos, organizados de acordo com o autor/ano de publicação, país/cidade, amostra e desempenho dos atributos da APS (adequados e inadequados).

\section{RESULTADOS}

O quadro 1 mostra a distribuição dos 34 estudos selecionados, todos com delineamento transversal, e os atributos classificados de acordo com a sua avaliação pelos profissionais de saúde da APS. A maioria dos estudos foram realizados no Brasil (85,3\%), e um estudo em cada um dos países: Uruguai, Paraguai, Chile, China e África do Sul.

Quanto à localização geográfica dos estudos realizados no Brasil, a maior parte se concentrou nas regiões sul e sudeste $(69 \%)$ e os demais nas regiões centro oeste e nordeste, esta última com menor número (apenas 4 trabalhos). Nenhum estudo foi encontrado na região norte do país.

A maioria dos estudos (67\%) tiveram como público alvo exclusivamente os profissionais de saúde e/ou gerentes da APS. Os demais estudos incluíram em sua amostra, além dos profissionais, usuários adultos ou cuidadores de crianças. Uma quantidade relevante dos estudos utilizou amostra composta por menos de 100 profissionais $(47,5 \%)$.

Quanto ao desempenho dos atributos da APS, o atributo acesso de primeiro contato foi o mais negativamente avaliado, tendo sido considerado adequado apenas nos estudos realizados na China e no Chile. Os atributos mais bem avaliados foram enfoque familiar $(79,4 \%)$, coordenação da atenção $(79,4 \%)$ e integralidade $(76,4 \%)$. 


\section{DISCUSSÃO}

Como pode ser observado, o PCATooL é um instrumento utilizado em diferentes países para avaliar a APS. Devido sua origem relativamente recente (STARFIELD, 2002), especialmente a versão brasileira destinada a profissionais (HAUSER et al., 2013), a maioria dos estudos com essa ferramenta são bem recentes.

Dentre os países que realizaram esse tipo de estudo, com profissionais de saúde, o Brasil foi o que mais publicou essas pesquisas, o que pode ser explicado pelo fato de ser um dos países cujo sistema de saúde é orientado pela APS. Além disso, o Ministério da Saúde publicou, em 2010, o Manual do Instrumento de Avaliação da APS (PCATool-BR), reforçando a importância da avaliação dos serviços de APS através dessa ferramenta, inclusive como um procedimento de rotina pelas equipes de saúde, de modo a institucionalizar a avaliação em saúde (BRASIL, 2010).

Estudos de revisões recentes evidenciaram uma quantidade notável de estudos nacionais e internacionais utilizando o PCATool, porém, nas versões voltadas para usuários e/ou cuidadores de crianças (DÁVILA et al., 2017; PRATES et al., 2017). Em se tratando de profissionais de saúde, ainda são escassos o número de estudos internacionais envolvendo essa população. Dentre os estudos internacionais, a China foi a que apresentou o maior número de participantes, com 401 profissionais da APS (ZOU et al., 2015). No Brasil, a maior amostra encontrada foi de 8.235 médicos cubanos do programa Mais Médicos, provenientes das cinco regiões brasileiras (KEMPER, 2019). No entanto, houve predominância de estudos com amostras limitadas e pouco representativas, resultando em estudos com baixo grau de evidência, comprometendo a qualidade das conclusões e comparação dos resultados. 
Quadro 1 - Distribuição dos estudos.

\begin{tabular}{|c|c|c|c|c|c|}
\hline $\mathbf{N}^{\circ}$ & Autor/ano & $\begin{array}{l}\text { País } \\
\text { Região } \\
\text { cidade }\end{array}$ & Amostra & $\begin{array}{l}\text { Desempenho } \\
\text { adequado }\end{array}$ & $\begin{array}{l}\text { Desempenho } \\
\text { inadequado }\end{array}$ \\
\hline 1 & $\begin{array}{l}\text { Rodríguez- } \\
\text { Rivero et al., } \\
2012\end{array}$ & $\begin{array}{l}\text { Paraguai, } \\
\text { Assunção }\end{array}$ & 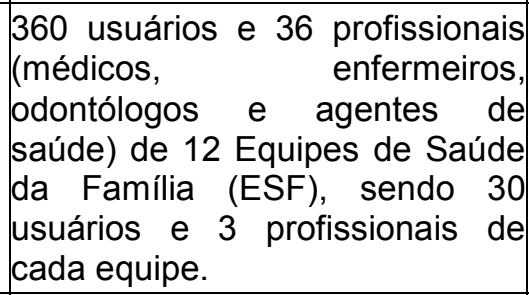 & $\begin{array}{l}\text { Longitudinalidad } \\
\text { e Integralidade } \\
\text { Coordenação } \\
\text { Orientação } \\
\text { Comunitária } \\
\text { Enfoque familiar }\end{array}$ & $\begin{array}{l}\text { Acessibilidade } \\
\text { de primeiro } \\
\text { contato }\end{array}$ \\
\hline 2 & $\begin{array}{l}\text { Berterretche } \\
\text { et al., } 2012\end{array}$ & $\begin{array}{l}\text { Uruguai, } \\
\text { Montevideo }\end{array}$ & $\begin{array}{l}178 \text { usuários, } 25 \text { profissionais e } \\
7 \text { gestores dos serviços da rede } \\
\text { de atenção de primeiro nível } \\
\text { (RAP). }\end{array}$ & \begin{tabular}{|l|} 
Longitudinalidad \\
e Integralidade \\
Coordenação \\
Orientação \\
comunitária \\
Enfoque familiar \\
\end{tabular} & $\begin{array}{l}\text { Acessibilidade } \\
\text { de primeiro } \\
\text { contato } \\
\text { Orientação } \\
\text { comunitária }\end{array}$ \\
\hline 3 & $\begin{array}{l}\text { Castro } \\
\text { al., } 2012\end{array}$ & $\begin{array}{l}\text { Brasil, Rio } \\
\text { Grande do Sul, } \\
\text { Porto Alegre }\end{array}$ & $\begin{array}{l}340 \text { profissionais (médicos e } \\
\text { enfermeiros) das unidades de } \\
\text { saúde amostradas nos diferentes } \\
\text { tipos de serviços públicos de } \\
\text { APS. }\end{array}$ & $\begin{array}{l}\text { Longitudinalidad } \\
\text { e Integralidade } \\
\text { Coordenação } \\
\text { Orientação } \\
\text { comunitária } \\
\text { Enfoque familiar }\end{array}$ & $\begin{array}{l}\text { Acessibilidade } \\
\text { de primeiro } \\
\text { contato }\end{array}$ \\
\hline 4 & $\begin{array}{l}\text { Vitoria et al., } \\
2013\end{array}$ & $\mid \begin{array}{l}\text { Brasil, Santa } \\
\text { Catarina, } \\
\text { Chapecó }\end{array}$ & $\begin{array}{l}98 \text { médicos e enfermeiros das } \\
\text { unidades básicas de saúde } \\
\text { (UBS) }\end{array}$ & $\begin{array}{l}\text { Integralidade } \\
\text { Coordenação } \\
\text { Orientação } \\
\text { comunitária } \\
\text { Enfoque familiar }\end{array}$ & $\begin{array}{l}\text { Acessibilidade } \\
\text { de primeiro } \\
\text { contato } \\
\text { Longitudinalidad } \\
\text { e }\end{array}$ \\
\hline 5 & $\begin{array}{l}\text { Chomatas } \\
\text { et al., } 2013\end{array}$ & $\begin{array}{l}\text { Brasil, Paraná, } \\
\text { Curitiba }\end{array}$ & $\begin{array}{l}490 \text { profissionais (médicos } \\
\text { pediatras, gineco-obstetras, } \\
\text { clínicos gerais e enfermeiros das } \\
\text { unidades tradicionais e médicos } \\
\text { generalistas, de família e } \\
\text { comunidade e enfermeiros das } \\
\text { unidades do tipo estratégia } \\
\text { saúde da família - ESF). }\end{array}$ & $\begin{array}{l}\text { Integralidade } \\
\text { longitudinalidade } \\
\text { Coordenação } \\
\text { Orientação } \\
\text { Comunitária } \\
\text { Enfoque familiar }\end{array}$ & $\begin{array}{l}\text { Acessibilidade } \\
\text { de primeiro } \\
\text { contato }\end{array}$ \\
\hline 6 & $\begin{array}{l}\text { Reis et al., } \\
2013\end{array}$ & \begin{tabular}{|l|} 
Brasil, \\
Maranhão, São \\
Luís
\end{tabular} & $\begin{array}{l}30 \text { gestores, } 80 \text { profissionais } \\
\text { (médicos e enfermeiros) e } 882 \\
\text { usuários da ESF. }\end{array}$ & & $\begin{array}{l}\text { Acessibilidade } \\
\text { de primeiro } \\
\text { contato }\end{array}$ \\
\hline 7 & $\begin{array}{l}\text { Alencar et } \\
\text { al., } 2014\end{array}$ & $\begin{array}{l}\text { Brasil, } \\
\text { Maranhão, São } \\
\text { Luís }\end{array}$ & $\begin{array}{l}32 \text { gestores, } 80 \text { profissionais } \\
\text { (médicos e enfermeiros) e } 883 \\
\text { usuários da ESF. }\end{array}$ & & $\begin{array}{l}\text { Enfoque familiar } \\
\text { Orientação } \\
\text { comunitária }\end{array}$ \\
\hline 8 & $\begin{array}{l}\text { Barbaro } \\
\text { al., } 2014\end{array}$ & Brasil, Interior & $\begin{array}{l}44 \text { profissionais (médicos e } \\
\text { enfermeiros) que atendem } \\
\text { adolescentes que estão } \\
\text { realizando pré-natal, de } \\
\text { diferentes unidades de APS. }\end{array}$ & \begin{tabular}{|l} 
Integralidade \\
longitudinalidade \\
Coordenação \\
Orientação \\
comunitária \\
Enfoque familiar \\
\end{tabular} & $\begin{array}{l}\text { Acessibilidade } \\
\text { de primeiro } \\
\text { contato }\end{array}$ \\
\hline 9 & $\begin{array}{l}\text { Silva et al., } \\
2014\end{array}$ & Brasil, Minas & $\begin{array}{l}34 \text { profissionais (médicos } \\
\text { enfermeiros) da ESF. }\end{array}$ & $\begin{array}{l}\text { Integralidade } \\
\text { longitudinalidade } \\
\text { enoordenação } \\
\text { Orientação } \\
\text { comunitária } \\
\text { Enfoque familiar } \\
\end{array}$ & $\begin{array}{l}\text { Acessibilidade } \\
\text { de primeiro } \\
\text { contato }\end{array}$ \\
\hline
\end{tabular}




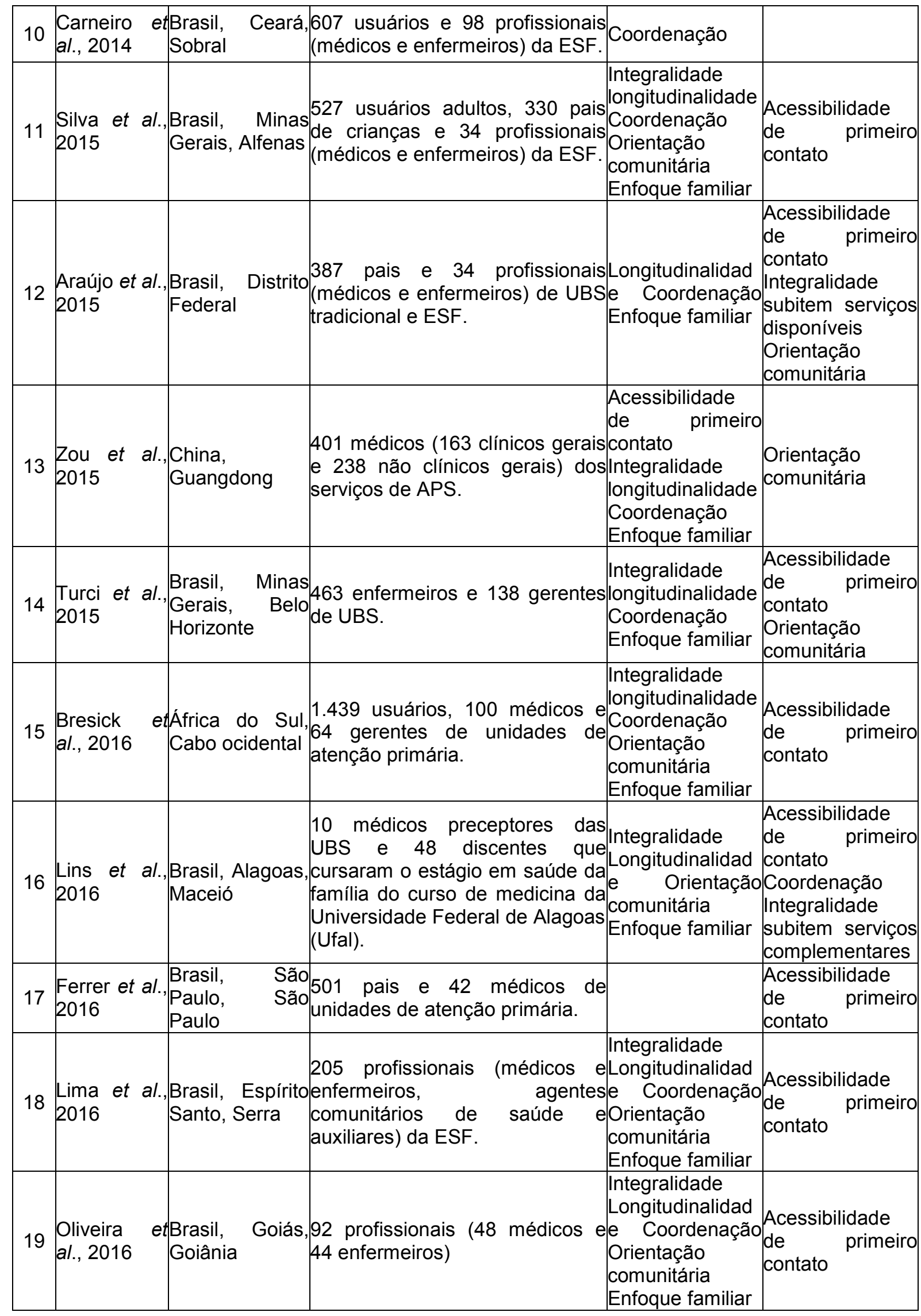




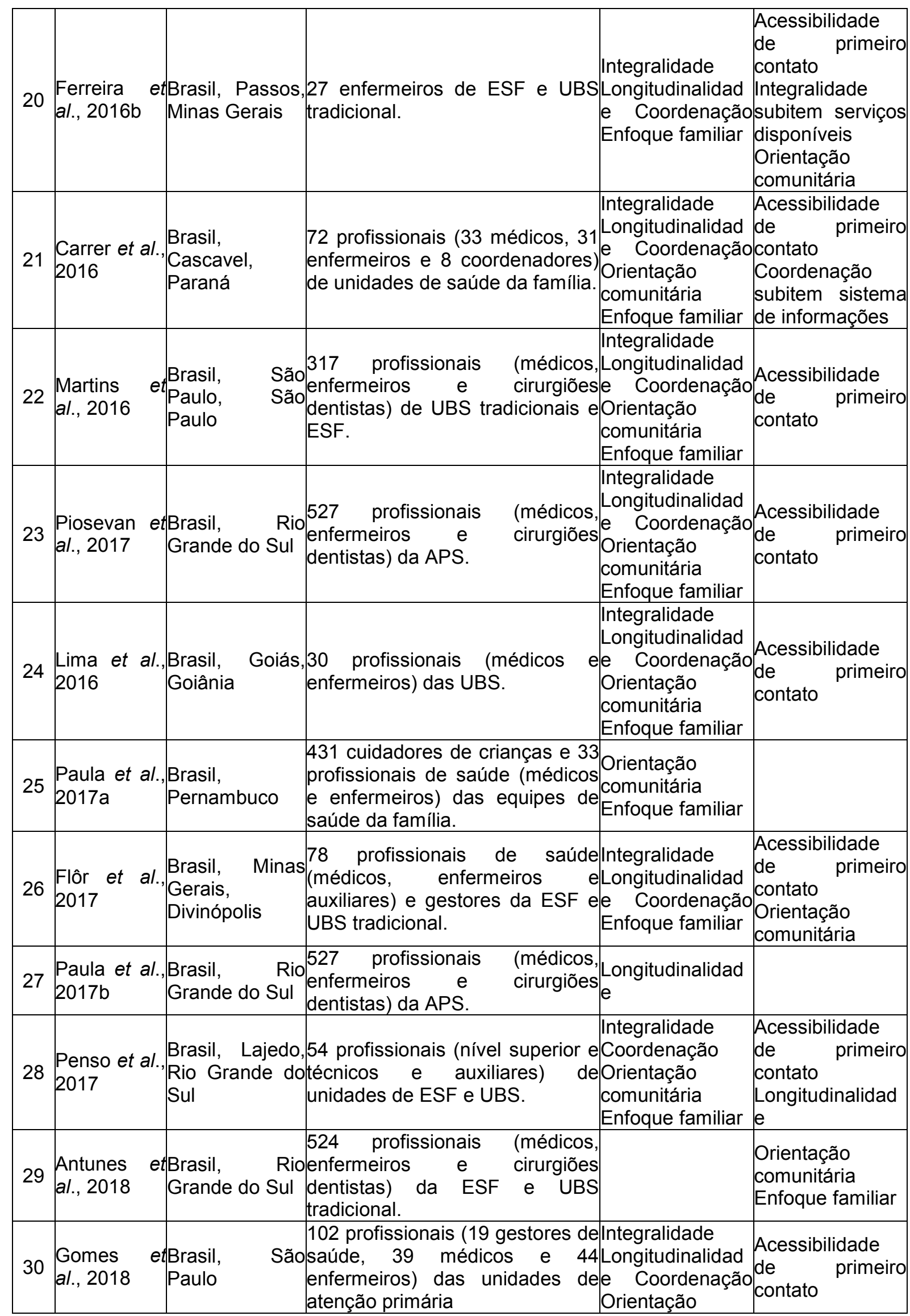




\begin{tabular}{|c|c|c|c|c|}
\hline & & & $\begin{array}{l}\text { Comunitária } \\
\text { Enfoque familiar }\end{array}$ & \\
\hline 31 & $\begin{array}{l}\text { Rolim et al., } \\
2019\end{array}$ & $\begin{array}{l}\text { Brasil, Ceará, } \\
\text { Fortaleza }\end{array}$ & 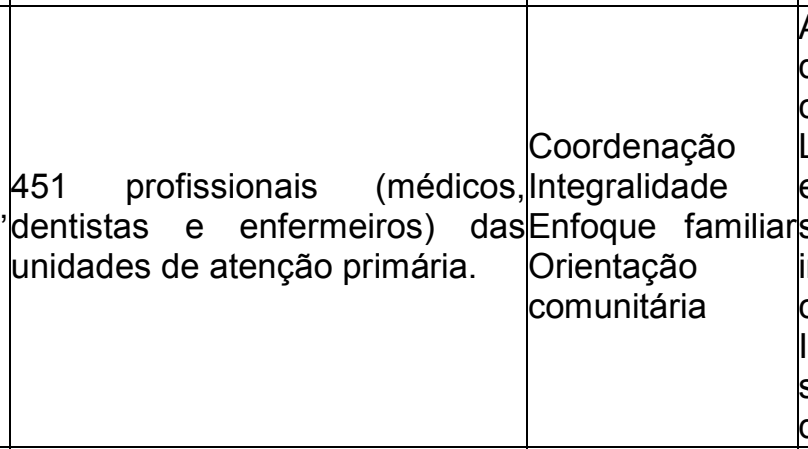 & $\begin{array}{l}\text { Acessibilidade } \\
\text { de primeiro } \\
\text { contato } \\
\text { Longitudinalidad } \\
\text { e Coordenação } \\
\text { rsubitem } \\
\text { integração de } \\
\text { cuidados } \\
\text { Integralidade } \\
\text { subitem serviços } \\
\text { disponíveis }\end{array}$ \\
\hline 32 & $\begin{array}{l}\text { Silva et al., } \\
2019\end{array}$ & \begin{tabular}{|l} 
Brasil, Minas \\
Gerais, \\
Diamantina
\end{tabular} & \begin{tabular}{|l|l|}
$707 \quad$ cuidadores e & \\
profissionais (médicos el & Lengitudinalidad \\
enfermeiros) das equipes de & Integralidadenação \\
atenção primária. & Enfoque familiar
\end{tabular} & $\begin{array}{l}\text { Acessibilidade } \\
\text { de primeiro } \\
\text { contato } \\
\text { Integralidade } \\
\text { subitem serviços } \\
\text { disponíveis } \\
\text { Orientação } \\
\text { comunitária }\end{array}$ \\
\hline 33 & $\begin{array}{l}\text { Kemper } \\
\text { al., } 2019\end{array}$ & Brasil & \begin{tabular}{|l|l|}
8.235 médicos cubanos do do Coordenação \\
programa Mais Médicos das \\
cinco regiões do país.
\end{tabular} & $\begin{array}{l}\text { Acessibilidade } \\
\text { de primeiro } \\
\text { contato }\end{array}$ \\
\hline 34 & $\begin{array}{l}\text { Pesse } \\
\text { Sorensen et } \\
\text { al., } 2019\end{array}$ & $\begin{array}{l}\text { Chile, } \\
\text { Santiago, } \\
\text { Conhalí }\end{array}$ & 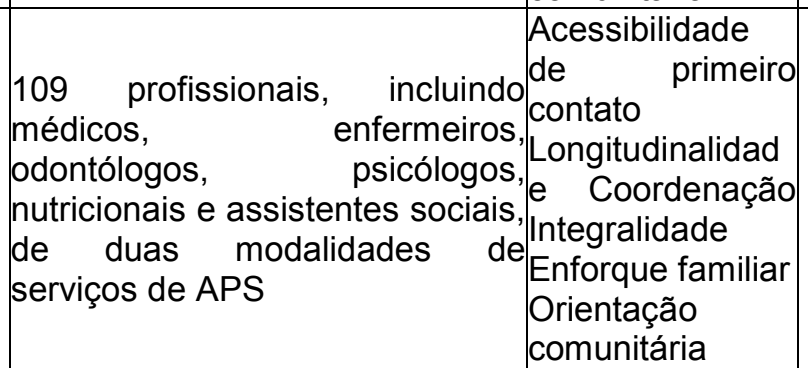 & \\
\hline
\end{tabular}

Em relação à avaliação dos atributos, somente dois estudos não avaliaram negativamente o atributo acessibilidade de primeiro contato, na China (ZOU et al., 2015) e no Chile (PESSE-SORENSEN et al., 2019). Considerando esse atributo como o acesso e utilização dos serviços como a primeira escolha do usuário para resolver um problema de saúde (STARFIELD, 2002), é preocupante que essa característica não esteja presente em diferentes regiões do Brasil e do mundo. Tratando-se do Sistema Único de Saúde (SUS), especificamente, a inadequação desse atributo compromete sobremaneira o princípio da universalidade.

No Brasil, alguns dos pontos relacionados a esse atributo podem ter contribuído para tal avaliação, tal como a inexistência da obrigatoriedade, na maioria das unidades, do funcionamento das unidades de APS aos sábados e domingos, 
bem como o serviço de telefonia disponível para o contato do usuário com o profissional de saúde. Apesar de não ser específica, a Política Nacional de Atenção Básica (PNAB) indica a organização dos serviços de acordo com as necessidades da população (BRASIL, 2017). É preciso repensar o distanciamento entre o colocado na PNAB e o que tem sido realizado como cultura institucional de funcionamento nesses serviços.

No cenário internacional, destaca-se aqui a África do Sul, um dos países que avaliou como inadequado o acesso. Esse país tem passado, nos últimos anos, por um processo de reforma para implantar o National Health Insurance, cujo um dos objetivos é aumentar o acesso da população aos serviços de saúde. Os aspectos históricos e sociais desse país e a atual elevada carga de doenças transmissíveis (ROA, 2015), refletem consideravelmente na avaliação de seus serviços.

A orientação comunitária foi o segundo atributo que recebeu pior avaliação ( $28,2 \%$ dos estudos), tendo sido o único atributo com tal avaliação na China (ZOU et al., 2015). Tal achado sinaliza para a necessidade de uma maior participação dos usuários nos processos de formulação de ações para melhoria da atenção, além da consideração das necessidades da comunidade no planejamento em saúde pelos profissionais.

Os atributos enfoque familiar, coordenação e integralidade foram os mais bem avaliados. Percebe-se, no entanto, que a avaliação desses atributos varia nas diferentes localidades do mundo, e mesmo entre as diversas regiões do Brasil. Essas disparidades podem ser explicadas pelos diferentes contextos culturais, sociais e econômicos dos diferentes países. No Brasil, a extensa dimensão territorial implica particularidades locais quanto a estrutura e organização dos serviços (PAIM, 2012).

O atributo longitudinalidade foi bem avaliado em mais da metade dos estudos. Esse atributo está relacionado com a familiaridade entre paciente e profissional, vínculo e satisfação do paciente (STARFIELD, 2002). No Brasil, características como a definição de uma população adscrita e a universalização do acesso à saúde expansão da APS, contribuem para o seu melhor desempenho (MENDES, 1996; LIMA, SOUSA, PRIMO, 2015). Em 5 estudos nacionais, entretanto, esse atributo foi mal avaliado. Mais uma vez, a inexistência de telefone para contato entre 
profissional e usuário na rotina das unidades tem influência negativa nesse resultado. Além disso, a alta rotatividade de profissionais, muito presente nesses serviços, dificulta a criação do vínculo com os usuários (LIMA et al., 2018).

Vale ressaltar algumas limitações encontradas nos artigos publicados: amostras pouco representativas na maioria dos estudos; o fato de apenas alguns dos atributos serem avaliados em determinadas pesquisas; e a falta de padronização quanto a classificação do desempenho dos atributos. Tais características dificultam a comparação dos dados entre as diferentes localidades, além de comprometerem a validade dos resultados encontrados. Apesar disso, estudos locais são considerados importantes e são incentivados pois seus resultados podem orientar mudanças significativas nos serviços de APS e reorientação do modelo de atenção.

\section{CONCLUSÃO}

Esta revisão apresentou um panorama geral da avaliação dos serviços de APS no mundo, utilizando o PCATool, versão profissionais. A maioria dos estudos obtiveram avaliação positiva, no geral, com exceção da acessibilidade de primeiro contato, cujo desempenho inadequado predominou em mais de $90 \%$ dos estudos, demonstrando que existe ainda um longo caminho a ser percorrido para o alcance de um modelo ideal de APS, visto que o acesso é indispensável para o alcance dos demais atributos.

Ademais, a orientação comunitária e a longitudinalidade foram o segundo e terceiro atributo, respectivamente, que mais apresentaram desempenho inadequado.

Verificou-se predominância por estudos realizados no Brasil, cujo desempenhos dos serviços demonstrou fragilidades, destacando-se a necessidade de fortalecimento da APS de acordo com os atributos estabelecidos no PCATool. 


\section{REFERÊNCIAS BIBLIOGRÁFICAS}

ALENCAR, M. N. et al. Avaliação do enfoque familiar e orientação para a comunidade na Estratégia Saúde da Família. Ciênc. Saúde Coletiva, Rio de Janeiro, v. 19, n. 2, p. 353-364, 2014.

ANTUNES, B. S.; PADOIN, S. M. de M.; PAULA, C. C. de. Avaliação dos atributos da atenção primária à saúde: criança e adolescente vivendo com HIV. Esc Anna Nery, Rio de Janeiro, v. 22, n 2: e20170233, 2018.

AOKI, T.; INOUE, M.; NAKAYAMA, T. Development and validation of the Japanese version of Primary Care Assessment Tool. Fam. Pract., Reino Unido, n. 33, v. 1, p. 112-117, 2016.

ARAUJO, R. L; MENDONCA, A. V. M.; SOUSA, M. F. Percepção dos usuários e profissionais de saúde no Distrito Federal: os atributos da atenção primária. Saúde debate, Rio de Janeiro, v. 39, n. 105, p. $387-399$.

BARBARO, M. C. et al. Prenatal Care for Adolescents and attributes of Primary Health Care.

Rev. Latino-Am. Enfermagem, v. 22, n. 1, p. 108-114, 2014.

BERRA S. et al. Validez y fiabilidad de la versión argentina del PCAT-AE para evaluar la atención primaria de salud. Rev. Panam. Salud Publica, Washington, US, v. 33, n. 1, p.30- 39, 2013.

BERRA, S. et al. Adaptación cultural y al sistema de salud argentino del conjunto de instrumentos para la evaluación de la Atención Primaria de la Salud. Rev. Argent. Salud Pública, Argentina, v. 2, n. 8, p. 6-14, 2011.

BERTERRETCHE, R.; SOLLAZZO, A. El abordaje de la Atención Primaria de Salud, modelos organizativos y prácticas: caso de un Centro de Salud público urbano de Montevideo, Uruguay 2011. Saúde em Debate, Rio de Janeiro, v. 36, n. 94, p. 461-472, 2012.

BRASIL, Ministério da Saúde. Portaria $n^{\circ} 2.436$, de 21 de setembro de 2017. Diário Oficial da União: seção 1, Brasília, DF, n. 163, p. 68, 22 set. 2017.

BRESICK, G. et al. Adaptation and crosscultural validation of the United States Primary Care Assessment Tool (expanded version) for use in South Africa. Afr J Prm Health Care Fam Med., South Africa, v.7, n.1, 2015.

BRESICK, G. et al. Adaptation and crosscultural validation of the United States Primary Care Assessment Tool (expanded version) for use in South Africa. Afr J Prm Health Care Fam Med., South Africa, v. 7, n. 1, 2016.

CARNEIRO, M. S. M. et al. Avaliação do atributo coordenação da Atenção Primária à Saúde: aplicação do PCATool a profissionais e usuários. Saúde Debate, Rio de Janeiro, v. 38, n.esp., p. 279-295, 2014.

CARRER, A. et al. Efetividade da Estratégia Saúde da Família em unidades com e sem Programa Mais Médicos em município no oeste do Paraná, Brasil. Ciênc. Saúde Coletiva, Rio de Janeiro, v. 21, n. 9, p. 2849-2860, 2016.

CASTRO, R. C. L. et al. Avaliação da qualidade da atenção primária pelos profissionais de saúde: comparação entre diferentes tipos de serviços. Cad. Saúde Pública, Rio de Janeiro, v. 28, n. 9, p. 1772-1784, 2012. 
CHOMATAS, E. et al. Avaliação da presença e extensão dos atributos da atenção primária em Curitiba. Rev Bras Med Fam Comunidade. Rio de Janeiro, v. 29, n. 8, p. 294-303, 2013.

D'AVILA, O.P. et al. O uso do Primary Care Assessment Tool (PCAT): uma revisão integrativa e proposta de atualização. Ciênc. Saúde Coletiva, Rio de Janeiro, v. 22, n. 3, p. 855-865, 2017.

FERREIRA, T. et al. Avaliação da qualidade da atenção primária à saúde de crianças e adolescentes com HIV: PCATool-Brasil. Rev Gaúcha Enferm, Porto Alegra, v. 37, n. 3: e61132, 2016b.

FERREIRA, V. D. et al. Avaliação dos atributos da Atenção Primária à Saúde em um Município Mineiro. Esc Anna Nery, Rio De Janeiro, v. 20, n. 4: e20160104, 2016a.

FLÔR, C. R. et al. Avaliação da atenção primária à saúde por profissionais de saúde: comparação entre modelo tradicional versus Estratégia Saúde da Família. Rev. Bras. Epidemiol., São Paulo, v. 20, n. 4, p. 714-726, 2017.

FRACOLLI, L. A. P. et al. Instrumentos de avaliação da Atenção Primária à Saúde: revisão de literatura e metassíntese. Ciên. Saúde Coletiva, Rio de Janeiro, v. 19, n.12, p.4851-60, 2014.

GOMES, M. F. P.; FRACOLLI, L. A. Avaliação da estratégia saúde da família sob a ótica dos profissionais. Rev Bras Promoç Saúde, Rio de Janeiro, v. 31, n. 3, p. 1-13, 2018.

HAGGERTY, J. L. et al. Room for improvement: patients' experiences of primary care in Quebec before major reforms. Can. Fam. Physician. Canada, v. 53, p. 1056-1057, 2007.

HARZHEIM, E. et al. Consistência interna e confiabilidade da versão em português do Instrumento de Avaliação da Atenção Primária (PCATool-Brasil) para serviços de saúde infantil. Cad Saúde Pública, Rio de Janeiro, v. 22, n. 8, p. 1649-1659, 2006.

HAUSER, L. et al. Tradução, adaptação, validade e medidas de fidedignidade do Instrumento de Avaliação da Atenção Primária à Saúde (PCATool-Brasil) no Brasil: versão profissionais de saúde. Rev. Bras. Med. Fam. Comunidade, Rio de Janeiro, v. 8 n. 29 p. 244-55, 2013.

LEE, J. H. et al. Development of the Korean primary care assessment tool--measuring user experience: tests of data quality and measurement performance. Int $\mathbf{J}$ Qual Health Care, Oxford, v. 21, n. 2, p. 103-111, 2009.

LIMA, E. F. A.; SOUSA, A. I.; PRIMO, C. C. Avaliação dos atributos da atenção primária na perspectiva das usuárias que vivenciam o cuidado. Rev. Latino-Am. Enfermagem, Ribeirão Preto, v. 23, n. 3, p.559-559, 2015.

LIMA, E. F. et al. Avaliação da Estratégia Saúde da Família na Perspectiva dos Profissionais de Saúde. Esc. Anna Nery, Rio de Janeiro, v. 20, n. 2, p. 275-80, 2016.

LIMA, L. G. et al. Atributos essenciais da Atenção Primária à Saúde: resultados nacionais do PMAQ-AB. Saúde Debate, Rio de Janeiro, v. 42, n. esp1, p.52-66, 2018.

LINS, T. S.; SOARES, F. J. P.; COELHO, J. A. P. M. Avaliação dos Atributos em Atenção Primária à Saúde no Estágio em Saúde da Família. Rev Bras Educ Med, Rio de Janeiro, v. 40, n. 3, p. 355-63, 2016.

MARTINS, S. J. et al. Estudo comparativo entre Unidades de Saúde com e sem Estratégia Saúde da Família por meio do PCATool. Rev Bras Med Fam Comunidade. Rio de Janeiro, v. 11, n. 38, p. 1-13, 2016.

MENDES, E.V. Uma agenda para a saúde. São Paulo: Hucitec; 1996. Na perspectiva dos 
gestores, profissionais e usuários. Ciênc. Saúde Coletiva, Rio de Janeiro, v. 18, n. 11, p. 33213331, 2013.

NASCIMENTO, L. do et al. Qualidade da Atenção Primária à Saúde de crianças e adolescentes vivendo com HIV. Rev Latino-Am Enfermagem, Ribeirão Preto, v. 24, n. e2720, p. 1-10, 2016.

OLIVEIRA, M.P. R.; MENEZES, I. H. C. F.; SOUSA, L. M.; PEIXOTO, M. R. G. Formação e qualificação de profissionais de saúde: fatores associados à qualidade da atenção primária. Rev Bras Educ Med, Rio de Janeiro, v. 40, n. 4, p. 547-59, 2016.

ORGANIZAÇÃO MUNDIAL DA SAÚDE/UNICEF (OMS/UNICEF). Cuidados primários de saúde: Relatório da Conferência Internacional sobre Cuidados Primários de Saúde, Alma- Ata, Rússia, 1978. Brasília: UNICEF, 1979.

PAIM, J. S. Atenção Primária à Saúde: uma receita para todas as estações? Saúde Debate, Rio de Janeiro, v. 36, n. 94, p.343-347, 2012.

PASARÍN, M. I. et al. An instrument to evaluate primary health care from the population perspective. Aten. Primaria, Espanha, v. 39, n.8, p. 395-401, 2007.

PAULA, C. C. et al. Atenção à saúde de crianças e adolescentes com HIV: avaliação da longitudinalidade. Acta Paul. Enferm., São Paulo, v. 30, n. 2, p. 136-43, 2017.

PAULA, C. C. et al. Atenção à saúde de crianças e adolescentes com HIV: avaliação da longitudinalidade. Acta Paul. Enferm. São Paulo, v. 30, n. 2, p. 136-43, $2017 \mathrm{~b}$.

PAULA, W. K. A. S. de et al. Orientação comunitária e enfoque familiar: avaliação de usuários e profissionais da estratégia saúde da família. Cad. Saúde Coletiva, Rio de Janeiro, v. 25, n. 2 , p. 242-248, 2017 a.

PENSO, J. M. et al. Avaliação da Atenção Primária à Saúde utilizando o Instrumento PCAToolBrasil. Rev Bras Med Fam Comunidade, Rio de Janeiro, v. 12, n. 39, p.1-9, 217.

PIOZEVAN, G. et al. Qualidade da atenção primária na perspectiva de profissionais: saúde de crianças e adolescentes com hiv. Texto Contexto Enferm, v. 26, n. 2e00180016, p. 1-11,2017.

PRATES, M. L. et al. Desempenho da Atenção Primária à Saúde segundo o instrumento PCATool: uma revisão sistemática. Ciênc. Saúde Coletiva, Rio de Janeiro, v. 22, n. 6, p.18811893, 2017.

REIS, R. S. et al. Acesso e utilização dos serviços na Estratégia Saúde da Família. Ciênc. saúde coletiva, Rio de Janeiro, v. 18, n.11, p.3321-3331, 2013.

RODRÍGUEZ-RIVEROS, M. I. et al. Evaluación de la Atención Primaria de Salud en un contexto urbano: percepción de actores involucrados - Bañado Sur - Paraguay, 2011. Saúde em Debate, Rio de Janeiro, v. 36, n. 94, p. 449-460, 2012.

ROLIM, L. B. et al. Avaliação dos atributos da Atenção Primaria à Saúde de Fortaleza, Ceará, Brasil. Rev Bras Enferm, v. 72, n. 1, p. 24-31, 2019.

SILVA, A. S.; BAITELO, T. C.; FRACOLLI, L. A. Avaliação da atenção primária à saúde: a visão de usuários e profissionais sobre a estratégia de saúde da família. Rev Latino-Am Enfermagem, Ribeirão Preto, v. 23, n. 5, p. 979-987, 2015.

SILVA, G. S.; ALVES, C. R. L. Avaliação do grau de implantação dos atributos da atenção primária à saúde como indicador da qualidade da assistência prestada às crianças. Cad. Saúde Pública, Rio de Janeiro, v. 35, n. 2: e00095418, 2019. 
SILVA, S. A.; NOGUEIRA, D. A.; PARAIZO, C. M. S., Avaliação da Atenção Primária à Saúde: visão dos profissionais de saúde. Rev Esc Enferm USP, São Paulo, v. 48, n. esp., p. 126- 132, 2014.

STARFIELD, B. Atenção primária: equilíbrio entre necessidades de saúde, serviços e tecnologia. ed. brasileira. Brasília: UNESCO, 2002. ISBN: 85-87853-72-4.

TSAI, J. et al. Physician specialty and the quality of medical care experiences in the context of the Taiwan national health insurance system. J Am. Board. Fam. Med., United States, v. 23, n. 3, p. 402-12, 2010a.

TSAI, J.; SHI, L.; YU, W. L.; LEBRUN, L. A. Usual source of care and the quality of medical care experiences: a cross-sectional survey of patients from a Taiwanese community. Med. Care, United States, v. 48, n. 7, p. 628-34, 2010.

TURCI, M. A.; COSTA, M. F. L.; MACINKO, J. Influências de fatores estruturais e organizacionais no desempenho da atenção primária à saúde em Belo Horizonte, Minas Gerais, Brasil, na avaliação de gestores e enfermeiros. Cad. Saúde Pública, Rio de Janeiro, v. 31, n. 9 , p.1941-1952, 2015.

VITORIA, A. M. et al. Avaliação dos atributos da atenção primária à saúde em Chapecó, Brasil. Rev Bras Med Fam Comunidade. Rio de Janeiro, v. 29, n. 8, p. 285-293, 2013.

WONG, S. Y. et al. Comparison of primary care experiences among adults in general outpatient clinics and private general practice clinics in Hong Kong. BMC Public Health, London, v. 10, p. $397,2010$.

ZOU, Y. et al. General practitioners versus other physicians in the quality of primary care: a cross-sectional study in Guangdong Province, China. BMC Family Practice. v.16:134, p. 1- 8, 2015. 